\title{
Perceções sobre o conceito de Dieta Mediterrânica e a sua evolução - perspetivas de profissionais do Algarve-Portugal
}

\section{Perceptions on the concept of Mediterranean diet and its evolution - perspectives of professionals from the Algarve-Portugal}

\author{
Helena Real ${ }^{a, b}$, Pedro Graça ${ }^{b}$ \\ ${ }^{a}$ Associação Portuguesa de Nutrição, Portugal \\ ${ }^{\text {b }}$ Faculdade de Ciências da Nutrição e Alimentação, Universidade do Porto, Portugal
}

\section{Resumo}

Introdução: Definir bem um conceito é algo importante para facilitar a transmissão de uma mensagem coerente e que perdure ao longo do tempo. Objetivos: Desenvolver um mapa conceptual da definição de Dieta Mediterrânica; avaliar as perceções sobre o conceito e evolução da Dieta Mediterrânica de personalidades representativas da Dieta Mediterrânica, do Algarve-Portugal. Metodologia: Foram aplicadas treze entrevistas semiestruturadas. $\mathrm{O}$ áudio foi gravado, transcrito, codificado tematicamente e as indicações dos entrevistados foram elencadas. A análise do conceito foi efetuada com base no método híbrido, em três fases: teórica, empírica e analítica. Resultados: Cruzando o conteúdo das entrevistas com a literatura desenvolveu-se um mapa conceptual da definição da Dieta Mediterrânica, com quatro eixos - alimentação, convivialidade, produção alimentar, estilo de vida - representando um modelo cultural, com impacto em áreas como ambiente, economia, saúde e turismo. Os entrevistados indicaram existir uma evolução do conceito de Dieta Mediterrânica, em consonância com a constante inovação tecnológica e de investigação, acautelando-se a desvirtuação do conceito e a sua preservação. Conclusões: Este trabalho apresenta, pela primeira vez, as perceções de profissionais portugueses sobre a definição e evolução da Dieta Mediterrânica, sendo o mapa conceptual um modelo inovador e potenciador de uma comunicação mais facilitada da sua definição.

Palavras-Chave: Conceito; Dieta Mediterrânica; Evolução; Mapa conceptual; Modelo híbrido.

\begin{abstract}
Introduction: Defining a concept well is important to facilitate the transmission of a coherent message that lasts over time. Objectives: To develop a conceptual map of the Mediterranean Diet definition; to evaluate perceptions about the concept and evolution of the Mediterranean Diet of personalities representative of the Mediterranean Diet, Algarve-Portugal. Methodology: Thirteen semi-structured interviews were applied. The audio was recorded, transcribed, thematically coded and the interviewees' indications were listed. The analysis of the concept was carried out based on the hybrid method, in three phases: theoretical, empirical and analytical. Results: Crossing the content of the interviews with the literature, a conceptual map of the definition of the Mediterranean Diet was developed with four axes - food, conviviality, food production, lifestyle - representing a cultural model, impacting on areas such as environment, economy, health and tourism. The interviewees indicated that there is an evolution of the Mediterranean Diet concept, in keeping with the constant technological and research innovation, taking care of the distortion of the concept and its preservation. Conclusions: This paper presents, for the first time, the perceptions of Portuguese professionals on the definition and evolution of the Mediterranean Diet, being the conceptual map an innovative model and a facilitator of the Mediterranean Diet definition communication.
\end{abstract}

Keywords: Concept; Mediterranean diet; Evolution; Conceptual map; Hybrid model. 


\section{Introdução}

E m 1975, Ancel Keys publicou, juntamente com a sua esposa Margaret, o livro "How to eat well and stay well. The Mediterranean way", onde apresenta os principais aspetos do padrão alimentar de populações que habitavam em diversas regiões da Grécia, Itália, França e Espanha (Keys \& Keys, 1975), fruto do desenvolvimento do Estudo dos Sete Países sobre alimentação e Doença Cardiovascular. Esta publicação surge após alguns anos de vivência deste casal em Pioppi, em Itália e do seu conhecimento da vida do Mediterrâneo. Apesar de este fisiologista americano ter iniciado os estudos sobre o Mediterrâneo na década de 50 , só mais tarde conseguiu alargar o conceito de alimentação Mediterrânica para algo mais abrangente. Em certa medida, isto poderá explicar o nome do livro acima indicado, após a publicação de "Eat well and stay well", em 1959, por querer passar a mensagem de que a Dieta Mediterrânica, como apelidou, não tinha apenas a ver com a ingestão de nutrientes mas também com uma forte ligação ao prazer de comer e vivenciar a alimentação de forma comunitária (Carmena, 2006).

A Dieta Mediterrânica representa mais do que apenas um modelo alimentar, englobando anos de história, tradições associadas e estilos de vida próprios da região. Desta forma, definir este conceito é algo complexo, o que justifica a existência de diferentes definições, com dimensões distintas.

A definição de um conceito é algo importante para facilitar a transmissão de uma mensagem coerente e que perdure ao longo do tempo. Se não for definido de forma consistente será mais difícil a sua operacionalização.
Desta forma, o desenvolvimento de um conceito é um processo que se traduz na clareza de ideias e termos para uma melhor perceção sobre o mesmo. Existem diversos modelos de análise e desenvolvimento de conceitos, sendo fundamental escolher o que melhor se adequar ao contexto em estudo.

Apesar de se estimar que a Dieta Mediterrânica se tenha vindo a construir na região do Mediterrâneo desde há 8000 anos sensivelmente, tendo em consideração os registos sobre os povos ao longo dos tempos (Altomare et al., 2013; Berry, Arnoni, \& Aviram, 2011), e sobre a estabilidade climática na região, neste artigo apenas se considerará o período após a criação da designação do conceito de Dieta Mediterrânica, por Ancel Keys, ou seja, a partir dos anos 50-60 do séc. XX.

\section{Objetivos}

Este estudo pretende desenvolver um mapa conceptual da definição de Dieta Mediterrânica e avaliar as perceções sobre o conceito e evolução da Dieta Mediterrânica de personalidades de diferentes áreas representativas da Dieta Mediterrânica, do Algarve-Portugal.

\section{Metodologia}

\section{Análise/desenvolvimento de conceito}

Para a análise/desenvolvimento de uma definição do conceito de Dieta Mediterrânica utilizou-se o método híbrido de Schwartz-Barcoot e Kim (Schwartz-Barcott, Patterson, Lusardi, \& Farmer, 2002), baseado em três fases: teórica, empírica e analítica (Figura 1). Foi ainda construído um mapa de conceitos (Figura 2), resultante da análise de dados efetuada.

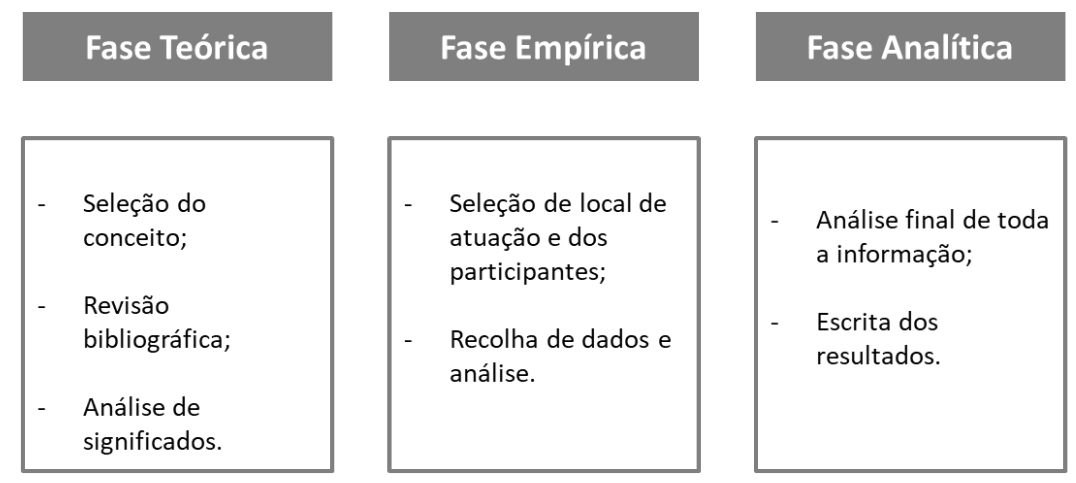

Figura 1. Fases componentes do método híbrido de Schwartz-Barcoot e Kim (Schwartz-Barcott et al., 2002). 


\section{Participantes}

Identificaram-se os potenciais participantes do estudo na zona do Algarve, onde se insere a comunidade representativa da inscrição na UNESCO da Dieta Mediterrânica em Portugal, correspondendo a profissionais com reconhecido conhecimento e experiência na área e que estivessem envolvidos na produção do conceito, por via do ensino e investigação (8 entrevistados com formação na área das Ciências da Nutrição, da Sociologia, da Biologia, da Arquelogia e da Agronomia), ou ligados à utilização do conceito, pela ligação ao poder local, turismo, hotelaria, saúde, cultura, agricultura (5 entrevistados com formação na área do Direito, do Turismo, da Hotelaria, das Ciências da Nutrição, da Sociologia e da Agronomia).

Relativamente ao consentimento informado por parte dos treze inquiridos e para o obter, foram inquiridos inicialmente todos os participantes por e-mail de forma a ser efetuado o convite e detalhar os procedimentos a seguir. Apresentaram-se de seguida as condições de participação, os passos para se manter a confidencialidade e informou-se sobre a gravação da entrevista, solicitando o consentimento devido. Todos os inquiridos receberam esta informação e, por escrito, responderam afirmativamente, concordando em participar no estudo nestas condições.

A taxa de adesão a este estudo foi de $81,3 \%$, tendo sido incluídos no estudo treze dos dezasseis participantes convidados.

\section{Recolha de dados}

Foi desenvolvido um guião de entrevista com questões que se distribuíam por três grupos: (1) perceção dos entrevistados sobre o conceito de Dieta Mediterrânica; (2) perceção sobre a evolução do conceito de Dieta Mediterrânica; e (3) possibilidades de utilização do conceito de Dieta Mediterrânica. Neste artigo serão explorados os aspetos ligados aos dois primeiros grupos, onde se inseriam questões como: qual a visão de cada entrevistado sobre o conceito de Dieta Mediterrânica; que vertentes (ou áreas) consideravam estarem incluídas no conceito da Dieta Mediterrânica; e se consideravam que o conceito da Dieta Mediterrânica evoluiu ao longo dos tempos e se sim, em que medida isso aconteceu; questionava-se ainda se consideravam que o conceito da Dieta Mediterrânica deve evoluir ou manter-se estanque ao longo dos tempos. Os dados referentes ao terceiro grupo foram já publicados recentemente (Helena Real \& Pedro Graça, 2019). As entrevistas decorreram entre maio e junho de 2017, tendo sido gravadas em formato áudio, mediante o consentimento dos entrevistados.

\section{Análise dos dados}

As entrevistas foram transcritas, anonimizadas e identificadas através de um código alfanumérico. Foi realizada a codificação temática, através do enquadramento do discurso dos entrevistados em categorias e subcategorias e da definição da unidade de registo e da unidade de contexto, para integrar a fase empírica do método híbrido na fase de análise/desenvolvimento do conceito de Dieta Mediterrânica e para a análise das perceções sobre a evolução do conceito.

\section{Resultados}

\section{Definição de conceito}

A aplicação do modelo híbrido para o desenvolvimento do conceito de Dieta Mediterrânica implicou três fases:

\section{Fase Teórica}

\section{a) Seleção e uso de conceito}

Neste estudo foi selecionado como objeto de análise a definição do conceito de Dieta Mediterrânica, definição que foi pela primeira vez cunhada por Ancel Keys (Moro, 2016). Desde que o mesmo iniciou a sua investigação nos anos 50 do século passado sobre a alimentação dos povos do Mediterrâneo que se assiste a uma proliferação na produção científica em torno da Dieta Mediterrânica, em várias áreas do saber, como a saúde, a antropologia, a economia, a agronomia, entre outras. Ao explorar este conceito na bibliografia é possível encontrar diversos termos que lhe são associados, que importa aprofundar. De seguida, são apresentadas as definições que poderão ser mais relevantes para elencar esses termos.

A primeira definição, proposta por Ancel Keys:

What is the Mediterranean Diet? One definition might be that it is what the Mediterranean natives eat. But as we know and think of it now, it is a relatively new invention. Tomatoes, potatoes, and beans, for example, came from America long after Christopher Columbus discovered the New World.[...] The heart of what we now consider the Mediterranean diet is mainly vegetarian: pasta in many forms, leaves sprinkled with olive oil, all kinds of vegetables in season, and often cheese, all finished off with fruit, and frequently washed down with wine (Keys, 1995).

A definição da UNESCO, que surgiu mais tarde, aquando a distinção de Património Cultural Imaterial da Humanidade:

The Mediterranean diet involves a set of skills, knowledge, rituals, symbols and traditions concerning crops, harvesting, fishing, animal husbandry, conservation, processing, cooking, and particularly the sharing and consumption of food. Eating together is the foundation of the cultural identity and continuity of communities throughout the Mediterranean basin. It is a moment of social exchange and communication, an affirmation and renewal of family, group or community identity. The Mediterranean diet emphasizes values of hospitality, neighbourliness, intercultural dialogue and creativity, and a way of life guided by respect for diversity. It plays a vital role in cultural spaces, festivals and celebrations, 
bringing together people of all ages, conditions and social classes. It includes the craftsmanship and production of traditional receptacles for the transport, preservation and consumption of food, including ceramic plates and glasses. Women play an important role in transmitting knowledge of the Mediterranean diet: they safeguard its techniques, respect seasonal rhythms and festive events, and transmit the values of the element to new generations. Markets also play a key role as spaces for cultivating and transmitting the Mediterranean diet during the daily practice of exchange, agreement and mutual respect (UNESCO, 2013).

E no contexto português, a definição apresentada em formato de princípios, apresentados aquando a distinção de Património Cultural Imaterial da Humanidade:

10 Princípios da Dieta Mediterrânica em Portugal

1. Frugalidade e cozinha simples que tem na sua base preparados que protegem os nutrientes, como as sopas, os cozidos, os ensopados e as caldeiradas;

2. Elevado consumo de produtos vegetais em detrimento do consumo de alimentos de origem animal, nomeadamente de produtos hortícolas, fruta, pão de qualidade e cereais pouco refinados, leguminosas secas e frescas, frutos secos e oleaginosas;

3. Consumo de produtos vegetais produzidos localmente, frescos e da época;

4. Consumo de azeite como principal fonte de gordura;

5. Consumo moderado de laticínios;

6. Utilização de ervas aromáticas para temperar em detrimento do sal;

7. Consumo frequente de pescado e baixo de carnes vermelhas;

8. Consumo baixo a moderado de vinho e apenas nas refeições principais;

9. Água como principal bebida ao longo do dia;

10. Convivialidade à volta da mesa".

Comissão responsável pela candidatura da Dieta Mediterrânica a Património Cultural Imaterial da Humanidade da UNESCO, 2013 (Barros, Carrageta, Graça, Queiroz, \& Sarmento, 2013)

Para além destas três definições apresentadas, foi ainda considerada uma reflexão sobre a definição de Dieta Mediterrânica de diversos especialistas da área, de diferentes pontos do mundo, publicada em formato de artigo, em 2014 (Trichopoulou et al., 2014).

Trichopoulou e colaboradores apontam que as definições de Dieta Mediterrânica podem variar consoante a geografia, o contexto histórico ou a nacionalidade dos autores, o que pode explicar as definições, por vezes, controversas, que se encontram na literatura (Trichopoulou et al., 2014).

O conceito de Dieta Mediterrânica é, atualmente, utilizado de forma bastante expressiva na literatura científica e não científica, neste caso, na imprensa, enquanto apresentação de modelo alimentar promotor de saúde. Por outro lado, é também utilizado por parte do setor agroalimentar, como potencial fator de valorização, perante o consumidor, das mensagens, dos produtos ou serviços veiculados pelas marcas.
De referir que o conceito de Ancel Keys (Keys, 1995) tenta caracterizar inicialmente um modo de comer, um padrão de consumo e que o conceito da UNESCO (UNESCO, 2013) tenta preservar este modo de consumo mas através da preservação do que permite a sua sobrevivência (a envolvente social, económica e cultural) e as suas formas de transmissão.

\section{b) Clarificação dos principais termos}

Os autores identificaram um conjunto de termos no contexto da análise das definições de Dieta Mediterrânica previamente elencadas.

- Dieta

A palavra "dieta" deriva do termo grego diaita, significando na sua génese "modo de vida" (Falcato \& Graça, 2015).

Este contexto implica uma predisposição do indivíduo para um comportamento prolongado no tempo, representando uma forma de se relacionar com a produção e consumo alimentar, à semelhança da população grega, determinando o seu dia-a-dia. A evolução da perceção deste termo mudou ao longo dos tempos, pelo que atualmente se verifica a associação do mesmo a restrição (Falcato \& Graça, 2015).

No caso da Dieta Mediterrânica, o termo "dieta" remeterá para "modo de vida" ou "estilo de vida", explorado mais à frente.

\section{- Mediterrânica}

O termo "Mediterrânica" refere-se ao que é do Mediterrâneo, apelando, assim, a uma condição geográfica. Trata-se de uma zona na base do Mar Mediterrâneo, onde crescem oliveiras, elementos essenciais da paisagem, e onde se destacam características relacionadas com o clima e a flora (Trichopoulou et al., 2014). A paisagem em si é algo característico desta zona geográfica, sendo bastante apreciada no contexto turístico, pelo que representa também um dos aspetos a usar na definição do conceito.

\section{- Alimentação}

Segundo a FAO (FAO, s.d.) o termo "alimentação" representa "qualquer substância, processada, semiprocessada ou crua, que se destine ao consumo humano, e inclui bebidas, pastilhas elásticas e qualquer substância que tenha sido usada na fabricação, preparação ou tratamento do "alimento", mas não inclui cosméticos ou tabaco ou substâncias usadas apenas como drogas" (FAO, s.d.).

Na literatura é possível encontrar diferentes definições para a parte alimentar da Dieta Mediterrânica. Contudo, as características alimentares mais comummente encontradas apontam para um modelo sobretudo vegetariano, com base na trilogia pão-vinho-azeite, sendo de destacar: elevada quantidade de azeite e 
azeitonas, fruta, hortícolas, cereais (sobretudo integrais), leguminosas e frutos oleaginosos, quantidade moderada de peixe e laticínios, e pequenas quantidades de carnes e os seus subprodutos. Vinho em moderação, durante as refeições e em consonância com as normas sociais e religiosas (Keys, 1980; Saura-Calixto \& Goni, 2009; Trichopoulou \& Lagiou, 1997; Trichopoulou et al., 2014; Willett et al., 1995). Será, assim um modelo maioritariamente à base de produtos vegetais (Trichopoulou et al., 2014).

Tendo em consideração a quantificação destes alimentos e os nutrientes ou outras substâncias que forneçam, Courtney Davis e colaboradores (Davis, Bryan, Hodgson, \& Murphy, 2015) propuseram, com base na literatura, que a componente alimentar da Dieta Mediterrânica incluísse 3-9 porções de hortícolas, $1 / 2-2$ porções de fruta, 1-13 porções de cereais e 8 ou mais porções de azeite. Esta distribuição conterá cerca de $9300 \mathrm{Kj} / 2214,3 \mathrm{Kcal}$ de energia, dos quais 37\% seriam gordura, $18,4 \%$ gordura monoinsaturada, $4,9 \%$ de gordura polinsaturada e $9 \%$ de gordura saturada. A relação Gordura moinsaturada: saturada será de 2,0, a ingestão de fibra de $33 \mathrm{~g}$ por dia, de vitamina $C$ de 225 $\mathrm{mg} /$ dia e folatos $508,2 \mu \mathrm{g} / \mathrm{dia}$.

Neste contexto, a frugalidade é algo inerente a estas recomendações, imperando assim a sugestão de um consumo moderado (Trichopoulou et al., 2014).

As informações quantitativas permitem ser usadas para a identificação do padrão alimentar mediterrânico, mediante uma abordagem a priori, com a formulação de índices de adesão (Bach et al., 2006), ou através de uma abordagem a posteriori, com recurso a técnicas estatísticas multivariadas, que analisam 0 consumo alimentar reportado pela população (Assi et al., 2016; Newby \& Tucker, 2004; Wirfalt, Drake, \& Wallstrom, 2013).

\section{- Ambiente}

O termo mais associado ao ambiente no contexto da alimentação é a sustentabilidade alimentar, nomeadamente as dietas sustentáveis. Em 2010, a FAO e a Bioversity International (FAO/Bioversity, 2010) organizaram um simpósio científico internacional sobre "biodiversidade e dietas sustentáveis", onde se obteve 0 consenso sobre a definição de dieta sustentável:

São as dietas com baixo impacto ambiental que contribuem para a segurança alimentar e para uma vida saudável para as gerações presentes e futuras. Dietas sustentáveis são protetoras e respeitadoras da biodiversidade e dos ecossistemas, culturalmente aceites, acessíveis, economicamente justas e baratas; são nutricionalmente adequadas, seguras e saudáveis; enquanto otimizam os recursos naturais e humanos.
A Dieta Mediterrânica, enquanto modelo de dieta sustentável, apresenta conceitos de biodiversidade, de ambiente, de economia, de saúde, de sazonalidade, de tradicionalidade dos alimentos, de consumo preferencial de produtos locais e frescos, e da utilização de cadeias curtas de distribuição (Burlingame \& Dernini, 2011).

\section{- Convivialidade}

A família é um elemento de excelência na Dieta Mediterrânica. À volta da mesa fazem-se as refeições, conversa-se, diverte-se e transmite-se ensinamentos entre gerações, onde a mulher assume um papel de realce (Barros et al., 2013; Moreno, 2015; Queiroz, 2015b; Valagão, 2015). Cozinhar, estar sentado em redor da mesa e partilhar comida no seio da família ou de amigos, promove um suporte social e a noção de comunidade (Serra-Majem, Bach-Faig, \& RaidoQuintana, 2012), onde imperam valores de hospitalidade ou vizinhança. Esta convivialidade, que permite trocas sociais e de comunicação, passa também para a comunidade, onde a Dieta Mediterrânica é dinamizada por Associações, confrarias e comissões de festas que permitem a organização de celebrações e festivais, onde a alimentação é o mote para o seu desenvolvimento e festividade (Barros et al., 2013; Queiroz, 2015a).

\section{- Cultura}

A Dieta Mediterrânica resulta de uma confluência de civilizações de diferentes continentes, cujas populações interagiram e se influenciaram ao longo dos tempos, através da sua fixação na área geográfica do Mediterrâneo. Deste processo resultou uma "cultura mediterrânica", fruto da diversidade de componentes alimentares, étnicas e religiosas, que se passou de geração para geração (Queiroz, 2015c). Assistiu-se a uma ritualização dos alimentos do Mediterrâneo, havendo uma forte relação entre os alimentos, a sua produção e as festividades e celebrações, muito comuns nesta região e que caracterizam os povos da mesma (Queiroz, 2015a). A importância dada a alimentos como o pão, o azeite e o vinho era transversal a várias religiões e também a costumes pagãos (Queiroz, 2015a), pelo que a harmonia entre os vários elementos também caracteriza a cultura destes povos.

Integrado no conceito de "Cultura", surge 0 "Património", como algo que herdamos do passado. Em 2003, a UNESCO aprovou a Convenção para a Salvaguarda do Património Cultural Imaterial, no sentido de promover uma proteção internacional do património cultural, de forma a proteger os sítios de valor universal excecional e a diversidade, que até então se destinava 
apenas ao património cultural tangível, móvel e imóvel (UNESCO, 2003). A Dieta Mediterrânica foi integrada na lista representativa do Património Cultural Imaterial da Humanidade precisamente com 0 objetivo de preservar e promover a transmissão desta herança às gerações futuras, de forma a recriarem-na, uma vez que representa um modelo cultural que rapidamente se perde se não for preservado e promovido (UNESCO, 2013).

\section{- Estilo de vida}

Na Infopédia (Infopédia, 2018) é possível ler sobre a definição de estilos de vida:

Prendem-se com práticas quotidianas e formas de consumo
que envolvem escolhas particulares e identitárias em
domínios tão díspares como a habitação, a alimentação,
os usos do corpo, o vestuário, a aparência, os hábitos de
trabalho, o lazer, a religião, a arte, a organização do espaço
e do tempo ou o convívio com os outros atores sociais.
Distintos e distintivos, com homologias ou correspondências
significativas a ligar as suas diversas componentes, os
estilos de vida tendem a configurar-se de forma coerente e
a exprimir as identidades pessoais e coletivas.

Mikael Jensen apresenta uma proposta de definição para estilo de vida, onde o identifica como um padrão de atos repetidos que são dinâmicos e, até certo ponto, ignorados pelo indivíduo, e que envolvem o uso de artefactos. Este estilo de vida é baseado em crenças sobre o mundo, e sua manutenção ao longo do tempo é orientada por intenções para atingir objetivos. Em outras palavras, um estilo de vida é um conjunto de hábitos que são direcionados pelo mesmo objetivo principal. É regulado por feedback (social) e pelo acesso a artefactos (Jensen, 2007).

Neste contexto, poderá ser inserido o descanso adequado reportado como característica dos Mediterrânicos (Keys \& Keys, 1975; Queiroz, 2015b), através de uma sesta diária (Naska, Oikonomou, Trichopoulou, Psaltopoulou, \& Trichopoulos, 2007); os hábitos culinários mais comuns, como as sopas, os guisados, os estufados, as açordas (Barros et al., 2013; Moreno, 2015; Valagão, 2015); a opção preferencial por produtos frescos, locais e da época (Barros et al., 2013; Keys, 1995; Moreno, 2015) e a atividade física regular (Keys \& Keys, 1975).

\section{- Produção Alimentar}

A componente da procura dos alimentos e/a sua produção são muito fortes nesta zona. Com base na agricultura, pesca e pecuária, é possível obter no Mediterrâneo uma diversidade interessante de alimentos. Sendo 0 solo fértil, conjugado com condições geoclimáticas favoráveis, é possível aceder a uma diversidade de espécies vegetais considerável, desde os cereais, aos produtos hortícolas, à fruta, às ervas aromáticas, aos frutos oleaginosos, ao azeite e ao vinho (Queiroz, 2015e, 2015f). O modelo de produção e colheita dos produtos respeita a biodiversidade, preserva os recursos naturais e aposta na sazonalidade, sendo, por isso, comummente apontado como um exemplo de sustentabilidade ambiental (Donini et al., 2016).

- Saúde

A Organização Mundial da Saúde define "Saúde" como "um estado de completo bem-estar físico, mental e social e não apenas a ausência de doença ou enfermidade" (WHO, 1946).

A Dieta Mediterrânica começou por ser associada à redução da incidência de doenças coronárias, tendo em conta o baixo conteúdo de gordura saturada, com base nos dados do Estudo dos "Sete Países" (Keys \& Keys, 1975). Depois desse estudo, vários foram os estudos que se multiplicaram, obtendo-se informação sobre a vantagem em se ter uma Dieta Mediterrânica face à diminuição do risco de diversas doenças: doenças cardiovasculares, obesidade, diabetes tipo 2, síndrome metabólica, doenças neurodegenerativas, cancro, relacionando-se com a longevidade (Martinez-Lacoba, Pardo-Garcia, Amo-Saus, \& Escribano-Sotos, 2018; Trichopoulou et al., 2014).

A necessidade de alteração de dieta é algo reportado desde há muito tempo, possivelmente desde o Estudo dos Sete Países de Ancel Keys (Keys \& Keys, 1975). Contudo, apesar do alerta e conhecimento gerado desde então, as escolhas alimentares vêm-se a deteriorar, o que se traduz num pior estado de saúde (Lang, 2013).

\section{Fase Empírica}

Foram administrados 13 questionários semiestruturados presenciais com uma média de 30,5 minutos de realização de cada entrevista, entre maio e junho de 2017.

\section{Fase Analítica}

Com base na revisão da literatura apresentada na fase teórica e no levantamento e caracterização das perceções sobre o conceito de Dieta Mediterrânica da fase empírica, foi possível efetuar um mapeamento de diferentes perceções do conceito de Dieta Mediterrânica que resume toda esta reflexão e que permitiu apresentar uma versão unificadora e os termos que poderão ser indicados quando se expõe este conceito (Figura 2). 


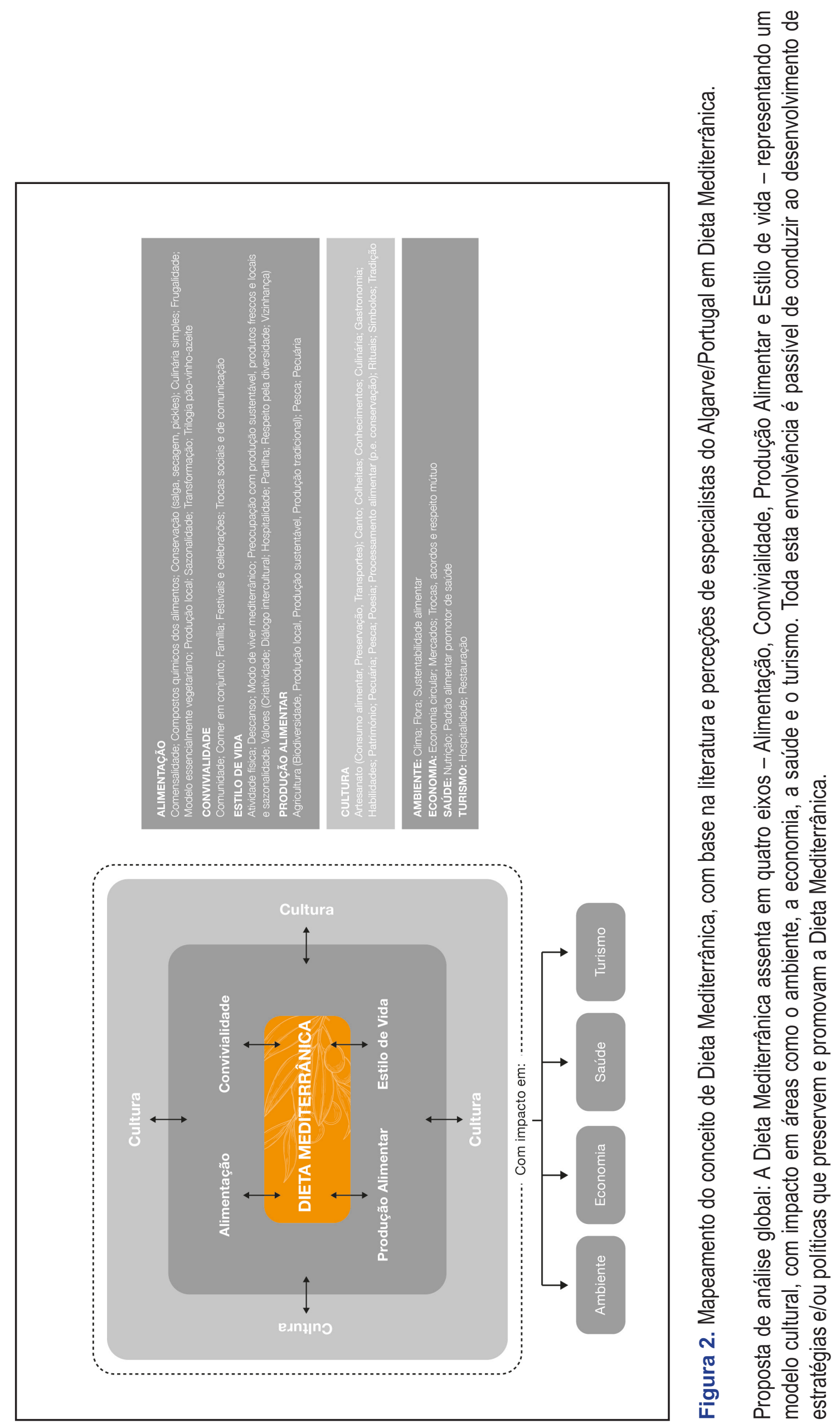




\section{Evolução do conceito}

Quando os entrevistados deste estudo foram questionados sobre a evolução do conceito de Dieta Mediterrânica, destacaram a importância da distinção como Património Cultural Imaterial da Humanidade pela UNESCO, como um dos principais marcos, referindo ainda que consideravam que o conceito não era estático, visto representar um modelo cultural e, por isso, dinâmico:

Estamos perante um conceito construído. Reconstruído, reinventado ao longo dos séculos. [ID 03]

0 conceito de Dieta Mediterrânica surgiu muito ligado à componente da saúde e a dimensão e a divulgação que o conceito de Dieta Mediterrânica teve desde o tempo de Ancel Keys, que foi quem trouxe o conceito para a ribalta, por assim dizer, está muito relacionado com a saúde, com a investigação científica na área da saúde, com a promoção da saúde e hábitos alimentares, saúde nas suas diferentes vertentes, saúde não só cardiovascular como foram os conceitos da investigação inicial, mas também saúde noutras vertentes, de combate à obesidade, prevenção do aparecimento de doenças crónicas metabólicas não transmissíveis na sua diversidade, de alimentação mediterrânica e saúde óssea, saúde mental. [ID 05]

A evolução fez com que o conceito aparecesse [....] temos a tecnologia que nos ajuda nessa evolução, claramente. [ID 01]

Eu acho que evoluiu claramente, quer dizer, o facto de termos alargado o número de disciplinas e de potenciais interessados na Dieta Mediterrânica trouxe um enriquecimento ao conceito porque contributos que surgiram de vários lados e o facto de, por vezes, as coisas poderem parecer contraditórias nalguns aspetos, porque nós, por exemplo, com o trabalho com a UNESCO não estamos muito voltados para as questões económicas. [ID 12]

Não é um conceito estanque. [ID 02]

Evoluiu [a Dieta Mediterrânica] porque a partir do momento em que ela começou a ser estudada como potencial promotor de saúde, se calhar houve até, não sei, uma apropriação do conceito de Dieta Mediterrânica para fazer valer outros pontos de vista e outras ideias [ID02].

Então evoluiu porque se adaptou àquilo que seriam também as recomendações científicas associadas à alimentação e a esse estilo de vida. [ID 02]

... passamos de uma visão extraordinariamente focada e redutora e minimalista de apenas e só prevenir as doenças cardiovasculares, [....] culminando aqui com uma visão muito social e cultural. [ID 07]

A cultura pode ser tudo menos ser estática. Isto é, não existe, ao contrário do que as pessoas podem pensar, um estatismo nas práticas cultuais, não há, as práticas culturais são extremamente dinâmicas e mais, estão em constante evolução e resultado das interações sociais, culturais, geográficas, etc. Portanto, há sempre uma evolução. [ID 08]

A candidatura a património imaterial da Humanidade veio ajudar, primeiro, a criar, a justificar o porquê da identidade mediterrânica e por outro lado gerar aqui um conjunto de informação para que pudesse ser dinamizado e replicado junto das pessoas em geral, inclusive do consumidor comum. [ID 13]

Este conceito patrimonializado é um conceito cultural, cultura, e a cultura é a cultura intangível, por isso é o património imaterial, é a cultura tangível, a agrícola, a piscicultura, a da conservação, tudo. [...] O conceito patrimonializado atualmente é o cultural. [ID 04]

Destacou-se também a oportunidade associada à distinção atribuída à Dieta Mediterrânica, para valorizar os recursos que o país tem e servir de motor para melhorar a sua promoção:

E, portanto, esta questão da Dieta Mediterrânica associada a vários países do Mediterrâneo e esta candidatura e este, digamos, Património Cultural Imaterial da Humanidade acho que veio também dar aqui um sentido às coisas completamente diferente, de uma sensibilidade diferente, um olhar para estas áreas de uma forma, enfim, no sentido de valorizarmos mais, percebermos mais 0 quanto é importante termos coisas ao pé que nem sempre sentimos e valorizamos e em função desta candidatura e desta aceitação, digamos, mundial também fez-nos aqui ter intervenções de mais responsabilidade, de mais exigência, mais parcerias envolvendo, enfim, as instituições de uma forma transversal, cada uma fazendo aquilo que tem a fazer, umas mais na ação direta, outras mais na promoção, outras mais na divulgação, outras mais no sentido da funcionalidade... [...] Portanto, sem artificialidade, sem fazermos aqui, digamos, mascararmos as coisas, maquilharmos as coisas e eu acho que nesse contexto acho que temos sido felizes e que temos aproveitado bem este processo, desta candidatura e desta valorização deste conceito, deste estilo de vida saudável que, enfim, a região tem procurado aproveitar, particularmente estes últimos 3 anos. [ID 09]

Todavia, foi também notado que a perceção de que o conceito seria passível de evolução poderia ser errada, sendo que o que evoluiria seria a sociedade, adaptandose o conceito a essas alterações:

Não é o conceito que evolui, é a sociedade que evolui, que impõe uma reflexão e uma reinterpretação. [ID 04]

O que nós podíamos era ver o que há de bom na tradição, porque as tradições são o nosso húmus cultural. Quer 
queira quer não, uma sociedade sem tradição não existe. E nós nascemos, caminhamos e avançamos, mesmo que fechemos os olhos, com esse húmus atrás de nós. Ora, vamos pegar nisso e vamos adaptar ao dia de hoje, de maneira engraçada, que se possa levar para a escola isto ou aquilo. [ID 04]

Ao questionar-se sobre a capacidade ou necessidade de evolução do conceito, os especialistas referem que é fundamental existir essa evolução, sobretudo adaptada à própria evolução da sociedade em que nos inserimos, de forma a melhor passar o conceito de Dieta Mediterrânica, sem que o mesmo se desvirtue ao longo do tempo:

...tudo isto é uma evolução, com os conceitos do passado, mas se calhar em proporções diferentes, de maneira diferente, e numa arquitetura dos diferentes setores. Portanto há uma evolução nítida. [ID 10]

As nossas necessidades atuais enquanto povo não são as mesmas necessidades que os nossos antepassados tinham e então este conceito de Dieta Mediterrânica tem que evoluir em função daquilo que é o nosso desenvolvimento [ID 13].

Tem que evoluir porque um conceito estanque é uma coisa que fica parada no tempo e morre, desaparece, não é? [...] E nós só podemos passar esse testemunho às gerações seguintes se nós soubermos adaptar a mensagem e 0 conceito e as práticas àquilo que é a sociedade atual, àquilo que são as exigências da sociedade atual, àquilo que são as características das gerações seguintes. [ID 05]

Se nós pegarmos nos grandes conceitos-chave, a frugalidade, no respeito pela produção sazonal dos bens alimentares, sejam eles quais forem e nos grandes grupos de alimentos e juntarmos a isso o modo de preparação simples, aquela cozinha que eu gosto de definir como sendo uma cozinha de água e tacho... depois se o tacho é de aço inoxidável ou de cobre como era no tempo dos nossos avós, acho que não faz diferença nenhuma [...] Faz diferença é a maneira como nós abordamos a prática do consumo de alimentos e a prática da preparação dos alimentos e a aquisição dos alimentos. [ID 06]

A Dieta Mediterrânica sendo um estilo de vida é uma prática cultural, um conjunto de práticas culturais, evolui necessariamente. Agora, não pode é perder a sua lógica, não pode perder a sua coerência. [ID 08]

...nós não temos que, à volta de um conceito e à volta de um perfil, à volta de um padrão gastronómico, estarmos reportados aos padrões de há 500 anos atrás. Agora, a ideia geral é que tem que se manter porque senão já não é a mesma coisa. [ID 11]

Foi ainda focada a mensagem de que a evolução do conceito deve ser acompanhada de forma a não se verificar a desvirtuação do conceito, no sentido de se ter algo completamente diferente da sua génese. Desta forma, atribui-se especial relevância aos planos de salvaguarda do conceito de Dieta Mediterrânica:

...porque quando se desvirtua o conceito criamos outra coisa qualquer. $\mathrm{E}$, por isso, é que o esforço da UNESCO é enorme nesta matéria, isto é, porque a UNESCO reduz-nos ou traz-nos para uma outra dimensão, que é a dimensão de que a história e a cultura ancestral deve ser preservada, que os atuais devem legar este ativo enorme de capital histórico e tradicional que deve ser preservado, registado, isto é, devemos ter um plano de salvaguarda para tudo isto e legar para o futuro. [ID 11]

\section{Discussão}

Identificar uma definição de Dieta Mediterrânica será fundamental para uma melhor reprodução e preservação do conceito. Courtney Davis, em 2015, publicou uma revisão da literatura sobre a definição de Dieta Mediterrânica, apontando-Ihe uma definição quantitativa de alimentos e grupos de alimentos, nutrientes e conteúdo em flavonoides (Davis et al., 2015). Poderá consultar-se na literatura várias formas de definir Dieta Mediterrânica: como um padrão alimentar, com base em guias alimentares, como a pirâmide, em sistemas a priori ou a posteriori de construção de padrões alimentares, com base em alimentos ou nutrientes, como modelo cultural ou como modelo de sustentabilidade (Bach-Faig et al., 2011; Davis et al., 2015; Donini et al., 2016; SauraCalixto \& Goni, 2009; Trichopoulou et al., 1995; Willett et al., 1995). No caso do uso dos modelos de avaliação da adesão é, assim, possível encontrar algumas discrepâncias nas definições, por via da utilização de diferentes critérios ou pontos de corte adotados (RaddVagenas, Kouris-Blazos, Singh, \& Flood, 2017; Real, Queiroz, \& Graca, 2019). Contudo, ao consultar a literatura é frequente verificar um especial enfoque sobre as características alimentares da Dieta Mediterrânica quando se faz a sua caracterização. Todavia, para além da alimentação existem outros fatores importantes que, em conjunto com questões alimentares, contribuem para os benefícios apontados, nomeadamente em termos de saúde e longevidade. Bellisle, em 2009, publicou um artigo onde referia precisamente alguns dos aspetos pouco destacados da Dieta Mediterrânica e que também influenciam o estado de saúde: a sesta, o número de refeições por dia, a presença ou ausência de lanches ou o horário das refeições (Bellisle, 2009).

De facto, a alimentação assume um papel central na vida social e cultural do Mediterrâneo, contudo, a definição de Dieta Mediterrânica vai mais além e assume-se como um modelo cultural complexo que reúne aspetos sociais 
e culturais que se interrelacionam em torno de sistemas alimentares e nunca de forma independente (Dernini \& Berry, 2015), o que poderá potenciar a sua transmissão entre gerações, mantendo os elementos estruturantes ao longo dos anos, apesar das alterações e evoluções sociais, comportamentais e históricas (Queiroz, 2015d).

O conceito de Dieta Mediterrânica apresentado por Ancel Keys (Keys, 1995) sublinhava uma relação nutriente-estado de saúde, com impacto positivo na saúde, mas que é relativamente robusta sob o ponto de vista da validação científica, visto ser algo relativamente fácil de avaliar e comprovar. Com a distinção da UNESCO, a definição inicial evoluiu para um conceito mais amplo onde se incorporam as questões culturais e sociais e onde a relação saúde-estilo de vida se tornam mais difíceis de estudar, já que as variáveis consideradas são menos definíveis e caracterizáveis. Além disso, não é fácil acumular evidência científica tão forte para estes elementos como a que obtemos com a componente alimentar da Dieta Mediterrânica.

O mapa de conceitos apresentado (Figura 2) resulta do somatório da consulta da literatura da área, daquilo que os entrevistados referiram e o que os investigadores consensualizaram sobre a matéria. Desta forma, se tivessem sido entrevistadas outras pessoas, eventualmente de outras zonas do país, o resultado poderia ser diferente. Um modelo conceptual permite apresentar os conceitos de forma integrada e dinâmica, não sendo fechado à possibilidade de se adaptar a forma de 0 apresentar à realidade que se tem, mediante a região ou o tipo de população. Além disso, deverá ser 0 mais transversal possível de forma a poder enquadrar a essência do conceito, mas ser permeável à evolução da sociedade onde o conceito se aplica, sem que se perca.

Da análise das perceções dos entrevistados deste estudo verificou-se que não existe uma ideia unificada sobre o que representa o conceito de Dieta Mediterrânica, o que é comum ao encontrado na literatura, como referido previamente. A distinção da Dieta Mediterrânica como Património Cultural Imaterial da Humanidade pela UNESCO veio redimensionar o conceito, relançando a discussão sobre o mesmo na comunidade científica e não científica. Começa-se, desta forma, a verificar uma desfragmentação do conceito inicial e uma redefinição do conceito de Dieta Mediterrânica, dado que é agora abordado e estudando cada vez por mais agentes, o que é indicativo da abrangência do conceito (Real \& Graça, 2019).

Desde a altura das investigações de Ancel Keys no Mediterrâneo, muitas foram as alterações que se verificaram ao longo dos tempos, devido a vários fatores, de onde se destaca a confluência de civilizações, as migrações e o turismo, o que permite uma transmissão de conhecimentos entre os povos, reinventando a sua forma de estar ao longo dos anos (Queiroz, 2015c). Além disso, também se pode considerar as alterações a nível da globalização dos sistemas de produção, acesso facilitado a alimentos de todo o mundo, bem como alteração de mentalidades e costumes, condicionados por fatores como a revolução reprodutiva, a emancipação da mulher na sociedade, a redução do tempo disponível para cozinhar, entre outros (CIHEAM, 2012). Todas estas vertentes podem ter efeito no estilo de vida, contribuindo para uma evolução a nível dos hábitos alimentares, verificando-se uma influência do Ocidente nos princípios da Dieta Mediterrânica, com consequente perda do equilíbrio alimentar característico desta Dieta.

A zona do Mediterrâneo sempre foi caracterizada pela introdução de novos alimentos, trazidos por outros povos, o que não contribuiu para o seu decréscimo de qualidade, pelo contrário, enriqueceu o portefólio de alimentos produzidos nesta zona do globo. Contudo, o que se assiste atualmente é a um crescimento de novos alimentos, sobretudo processados, e com conteúdos de açúcar, sal e/ou gorduras desproporcionais, promovendo um consumo frequente nos mesmos, desequilibrando a balança. Uma projeção da FAO para 2030 prevê o crescimento da oferta de produtos de origem animal e alimentos mais densos em termos energéticos (Alexandratos, 2006). Esta previsão, associada ao rápido urbanismo, aumento dos rendimentos da população, alterações tecnológicas no setor agroalimentar, disseminação de venda de comida económica e pobre em termos nutricionais e globalização podem por em questão a adesão à Dieta Mediterrânica (Alexandratos, 2006).

Dados de uma publicação recente apontam para uma estimativa de redução da adesão à Dieta Mediterrânica entre 1961-65 e 2000-03. Entre 2004-2011 estima-se uma estabilização da adesão, sendo que em 16 dos 41 países selecionados para o estudo, da latitude $40^{\circ}$ Norte (países do Mediterrâneo, da Ásia, Japão, América do Norte e Península Ibérica) estima-se um aumento de adesão. Os países do Mediterrâneo apresentaram especial deterioração da adesão (Vilarnau et al., 2018).

Todavia, o património, não só alimentar, mas também cultural associado à Dieta Mediterrânica é passível de ser preservado, daí a distinção da UNESCO, onde Portugal se inseriu, em 2013 (Alexandratos, 2006; UNESCO, 2013). Esta distinção permitirá desenvolver formas 
de preservar o conceito de Dieta Mediterrânica face às constantes evoluções que a sociedade apresenta. Quando se questionou sobre a eventual evolução do conceito de Dieta Mediterrânica, diversas foram as respostas sobre essa possibilidade ou o dinamismo que pode existir em redor da evolução da Dieta Mediterrânica. Contudo, um dos entrevistados referiu que a evolução a registar será a da sociedade e não a do conceito da Dieta Mediterrânica. A apresentação de diferentes pontos de vista sobre questões idênticas pode dever-se ao facto de a maior parte dos estudos e reflexões sobre Dieta Mediterrânica em Portugal terem ocorrido após a distinção da UNESCO, o conhecimento em Portugal é ainda pouco sólido, estando em clara fase de crescimento e sedimentação. Estima-se que o conhecimento sobre 0 assunto seja sobretudo proveniente da aplicação do conceito, e, por isso, muito mais utilitarista e menos assente numa discussão conceptual e a partir das suas premissas históricas.

A evolução da sociedade induz a inclusão de novos alimentos, novas técnicas, novas tecnologias e novos estilos de vida. 0 desafio será garantir que esta evolução não desvirtue os princípios originais da Dieta Mediterrânica. Será, portanto, importante promover a transmissão de memórias de saberes, cheiros e sabores entre gerações, integrando estes conhecimentos ancestrais na contemporaneidade (Queiroz, 2015d). 0 modelo de consumo alimentar investigado por Ancel Keys será facilmente adaptado ao longo dos anos. Contudo, a tradicionalidade de determinados fatores pode perderse com a evolução da sociedade, daí a importância da UNESCO neste contexto, por tentar preservar o modelo como um todo. Para se proteger é necessário trabalhar uma definição clara do conceito. Contudo, no caso da Dieta Mediterrânica assistimos a uma definição bastante abrangente e que ainda se mantém em sedimentação em termos de conhecimento sobre a mesma, o que dificulta a capacidade de ser preservada e promovida às novas gerações.

Atualmente, o Mediterrâneo está também a passar por uma transição nutricional, onde coexistem problemas de desnutrição e obesidade e outras doenças crónicas não transmissíveis. As alterações alimentares ocorridas poderão dever-se a um aumento rápido da população, globalização e urbanização dos países desta região (Dernini et al., 2013).

Por outro lado, será importante perceber que pode existir uma definição de conceito transversal a todos os países do Mediterrâneo, mas que a sua aplicação poderá ser distinta em cada país, com base nas especificidades de cada um (Dernini \& Berry, 2015). Nos países do Mediterrâneo as tradições alimentares mudam de país para país (Trichopoulou, Soukara, \& Vasilopoulou, 2007), tendo em conta as diferentes realidades de cada um a nível cultural, religioso, climatérico, económico ou social, emergindo, contudo, um traço comum que permite realçar aquilo que se reconhece como Dieta Mediterrânica, ou seja, a essência do seu conceito. Tendo em consideração todas as mudanças da sociedade, não se torna fácil implementar um modelo conceptual da Dieta Mediterrânica, pelo que é fundamental a sua discussão e promoção, sobretudo junto dos mais jovens. Neste contexto, foi desenvolvido um mapa conceptual da definição de Dieta Mediterrânica, com base nas perceções de um conjunto de personalidades de diferentes áreas representativas da Dieta Mediterrânica, do Algarve-Portugal, e avaliou-se as suas perceções sobre o conceito e evolução da Dieta Mediterrânica, sendo que esperamos que constituam ferramentas e informação importantes no processo de reflexão sobre a temática.

\section{Conclusões}

Neste artigo foi apresentada uma análise extensiva do conceito de Dieta Mediterrânica, propondo-se a apresentação de um mapa conceptual, que realça a abrangência do conceito, colocando de parte a associação mais redutora exclusivamente à alimentação e saúde. Além disso, a sistematização da classificação do conceito de Dieta Mediterrânica num mapa assumese como um modelo inovador que permitirá certamente uma comunicação mais facilitada da sua definição.

A perceção da Dieta Mediterrânica associada à cultura e a termos difusos e variados traz oportunidades, mas também algumas dificuldades a quem quer utilizar pedagogicamente o conceito. A ligação do conceito à saúde permitia uma mais fácil transmissão do mesmo, contudo, tornava-se incompleto. Neste sentido, será expectável que várias áreas do saber se interligueme criem sinergias neste contexto de passagem da mensagem à população, nomeadamente às crianças, visto ser uma das formas mais apontadas para a necessária promoção e preservação da Dieta Mediterrânica. Também já o próprio Ancel Keys abordava a importância fulcral das crianças na passagem da mensagem da Dieta Mediterrânica aos seus pais (Keys, 1995).

A distinção da UNESCO como Património Cultural Imaterial da Humanidade veio consolidar a Dieta Mediterrânica como modelo cultural e dinâmico que deve ser preservado no contexto de uma sociedade que evolui 
ao longo dos tempos, de forma a garantir a salvaguarda das premissas básicas do conceito para que este não se desvirtue no decorrer da evolução.

\section{Referências Bibliográficas}

CIHEAM. (2012). Mediterra 2012. The Mediterranean Diet for Sustainable Regional Development. Paris: Presses de Sciences Po.

Alexandratos, N. (2006). The Mediterranean diet in a world context. Public Health Nutrition, 9(1a), 111-117.

Altomare, R., Cacciabaudo, F., Damiano, G., Palumbo, V. D., Gioviale, M. C., Bellavia, M., ... Lo Monte, A. I. (2013). The mediterranean diet: a history of health. Iran Journal of Public Health, 42(5), 449-457.

Assi, N., Moskal, A., Slimani, N., Viallon, V., Chajes, V., Freisling, H., . . . Ferrari, P. (2016). A treelet transform analysis to relate nutrient patterns to the risk of hormonal receptor-defined breast cancer in the European Prospective Investigation into Cancer and Nutrition (EPIC). Public Health Nutrition, 19(2), 242-254. doi:10.1017/s1368980015000294.

Bach-Faig, A., Berry, E. M., Lairon, D., Reguant, J., Trichopoulou, A., Dernini, S., . . Serra-Majem, L. (2011). Mediterranean diet pyramid today. Science and cultural updates. Public Health Nutrition, 14(12a), 2274-2284. doi:10.1017/s1368980011002515.

Bach, A., Serra-Majem, L., Carrasco, J. L., Roman, B., Ngo, J., Bertomeu, I., \& Obrador, B. (2006). The use of indexes evaluating the adherence to the Mediterranean diet in epidemiological studies: a review. Public Health Nutrition, 9(1a), 132-146.

Barros, V., Carrageta, M., Graça, P., Queiroz, J., \& Sarmento, M. (2013). Dieta Mediterrânica - Um património civilizacional partilhado. Retrieved from Tavira: https://comum.rcaap.pt/ bitstream/10400.26/10480/1/6927.pdf.

Bellisle, F. (2009). Infrequently asked questions about the Mediterranean diet. Public Health Nutrition, 12(9a), 1644-1647. doi:10.1017/s1368980009990498.

Berry, E. M., Arnoni, Y., \& Aviram, M. (2011). The Middle Eastern and biblical origins of the Mediterranean diet. Public Health Nutrition, 14(12a), 2288-2295. doi:10.1017/s1368980011002539.

Burlingame, B., \& Dernini, S. (2011). Sustainable diets: the Mediterranean diet as an example. Public Health Nutrition, 14(12a), 2285-2287. doi:10.1017/ s1368980011002527.

Carmena, R. (2006). Nota Biográfica del Dr. Ancel Keys. In P. y. A. Mediterranean Diet Foundation y Ministerio de Agricultura (Ed.), Comer bien, sentirse bien. La receta Mediterránea. Barcelona.

Davis, C., Bryan, J., Hodgson, J., \& Murphy, K. (2015). Definition of the Mediterranean Diet; a Literature Review. Nutrients, 7(11), 9139-9153. doi:10.3390/ nu7115459.

Dernini, S., \& Berry, E. M. (2015). Mediterranean Diet: From a Healthy Diet to a Sustainable Dietary Pattern. Frontiers in Nutrition, 2, 15. doi:10.3389/ fnut.2015.00015.

Dernini, S., Meybeck, A., Burlingame, B., Gitz, V., Lacirignola, C., Debs, P., . . . El Bilali, H. (2013). Developing a methodological approach for assessing the sustainabitity of diets: The Mediterranean diet as a case study. New Medit, 12(3), 28-36.

Donini, L. M., Dernini, S., Lairon, D., Serra-Majem, L., Amiot, M. J., Del Balzo, V., . . Berry, E. M. (2016). A Consensus Proposal for Nutritional Indicators to Assess the Sustainability of a Healthy Diet: The Mediterranean Diet as a Case Study. Frontiers Nutrition, 3, 37. doi:10.3389/fnut.2016.00037.

Falcato, J., \& Graça, P. (2015). A Evolução Etimológica e Cultural do Termo "Dieta". Revista Nutrícias, 24, 12-15.

FAO. Definitions for the purposes of the Codex Alimentarius. Retrieved from http://www.fao.org/ docrep/005/Y2200E/y2200e07.htm.

FAO/Bioversity. (2010). Report of the international symposium on Biodiversity and Sustainable Diets. Retrieved from Rome: http://www.fao.org/ag/humannutrition/285060efe4aed57af34e2dbb8dc578d465df8b.pdf.

Infopédia. (2018). Estilos de vida in Artigos de apoio Infopédia. 2003-2018. Retrieved from https://www. infopedia.pt/apoio/artigos/\$estilos-de-vida.

Jensen, M. (2007). Defining lifestyle. Environmental Sciences, 4(2),63-73.doi:10.1080/15693430701472747.

Keys, A. (1980). Seven countries: a multivariate 
analysis of death and coronary heart disease. Cambridge: Harvard University Press.

Keys, A. (1995). Mediterranean diet and public health: personal reflections. American Journal of Clinical Nutrition, 61(6 Suppl), 1321s-1323s.

Keys, A., \& Keys, M. (1975). How to eat well and stay well. The Mediterranean way. New York, USA: Doubleday Publishing.

Lang, T. (2013). Alimentos sustentáveis para dietas sustentáveis? O desafio da saúde pública ecológica. In F. C. Gulbenkian (Ed.), O Futuro da Alimentação: ambiente, saúde e economia (pp. 247).

Martinez-Lacoba, R., Pardo-Garcia, I., Amo-Saus, E., \& Escribano-Sotos, F. (2018). Mediterranean diet and health outcomes: a systematic meta-review. European Journal of Public Health, 28(5), 955961. doi:10.1093/eurpub/cky113.

Moreno, I. (2015). Culturas Mediterrânicas e sistemas alimentares: continuidades, imaginários e novos desafios. In U. d. Algarve (Ed.), Dimensões da Dieta Mediterrânica: Património Cultural Imaterial da Humanidade (pp. 301).

Moro, E. (2016). The Mediterranean Diet from Ancel Keys to the UNESCO Cultural Heritage. A Pattern of Sustainable Development between Myth and Reality. Procedia - Social and Behavioral Sciences, 223(Supplement C), 655-661. doi:https:// doi.org/10.1016/j.sbspro.2016.05.380.

Naska, A., Oikonomou, E., Trichopoulou, A., Psaltopoulou, T., \& Trichopoulos, D. (2007). Siesta in healthy adults and coronary mortality in the general population. Archives of Internal Medicine, 167(3), 296-301. doi:10.1001/archinte.167.3.296.

Newby, P. K., \& Tucker, K. L. (2004). Empirically derived eating patterns using factor or cluster analysis: a review. Nutrition Reviews, 62(5), 177-203.

Queiroz, J. (2015a). Alimentos sagrados e festividades cíclicas. In Althum.com (Ed.), Dieta Mediterrânica: uma herança milenar para a humanidade $\left(2^{\mathrm{a}} \mathrm{ed}\right.$., pp. 255). Lisboa.

Queiroz, J. (2015b). Diaita, um estilo de vida. In Althum. com (Ed.), Dieta Mediterrânica: uma herança milenar para a humanidade ( $2^{a}$ ed., pp. 255). Lisboa.
Queiroz, J. (2015c). O Doutor "colesterol": Ancel Keys e a "descoberta" da Dieta Mediterrânica. In Althum.com (Ed.), Dieta Mediterrânica. Uma herança milenar para a Humanidade (2a ed., pp. 255). Lisboa.

Queiroz, J. (2015d). O padrão alimentar da Dieta Mediterrânica Dieta Mediterrânica. Uma herança milenar para a humanidade $\left(2^{\mathrm{a}} \mathrm{ed}\right.$., $\mathrm{pp}$. 255). Lisboa: Althum.com.

Queiroz, J. (2015e). Ouro líquido do Mediterrâneo: 0 azeite. In Althum.com (Ed.), Dieta Mediterrânica. Uma herança milenar para a humanidade $\left(2^{\mathrm{a}}\right.$ ed., pp. 255). Lisboa.

Queiroz, J. (2015f). A vinha e o néctar dos Deuses. In Althum.com (Ed.), Dieta Mediterrânica. Uma herança milenar para a humanidade $\left(2^{\mathrm{a}}\right.$ ed., $\mathrm{pp}$. 255). Lisboa.

Radd-Vagenas, S., Kouris-Blazos, A., Singh, M. F., \& Flood, V. M. (2017). Evolution of Mediterranean diets and cuisine: concepts and definitions. Asia Pacific Journal of Clinical Nutrition, 26(5), 749-763. doi:10.6133/apjcn.082016.06.

Real, H., \& Graça, P. (2019). Marcos da História da Dieta Mediterrânica, desde Ancel Keys. Acta Portuguesa de Nutrição, 17, 6-14.

Real, H., \& Graça, P. (2019). Perceções de utilização do conceito de Dieta Mediterrânica, potencial utilização indevida e perspetivas a explorar $=$ Perceptions of use of the concept of Mediterranean diet, potential misuse and prospects to explore. Revista Española de Comunicación en Salud, 10(2), 147-159.

Real, H., Queiroz, J., \& Graca, P. (2019). Mediterranean food pattern vs. Mediterranean diet: a necessary approach? International Journal of Food Science Nutrition, 1-12. doi:10.1080/09637486.2019.1617838.

Saura-Calixto, F., \& Goni, I. (2009). Definition of the Mediterranean diet based on bioactive compounds. Critical Reviews in Food Science and Nutrition, 49(2), 145-152. doi:10.1080/10408390701764732.

Schwartz-Barcott, D., Patterson, B. J., Lusardi, P., \& Farmer, B. C. (2002). From practice to theory: tightening the link via three fieldwork strategies. Journal of Advanced Nursing, 39(3), 281-289.

Serra-Majem, L., Bach-Faig, A., \& Raido-Quintana, B. (2012). Nutritional and cultural aspects of the 
Mediterranean diet. International Journal of Vitamin and Nutrition Research, 82(3), 157-162. doi:10.1024/0300-9831/a000106.

Trichopoulou, A., Kouris-Blazos, A., Wahlqvist, M. L., Gnardellis, C., Lagiou, P., Polychronopoulos, E., . . . Trichopoulos, D. (1995). Diet and overall survival in elderly people. British Medical Journal, 311(7018), 1457-1460.

Trichopoulou, A., \& Lagiou, P. (1997). Healthy traditional Mediterranean diet: an expression of culture, history, and lifestyle. Nutrition Reviews, 55(11 Pt 1), 383-389.

Trichopoulou, A., Martínez-González, M. A., Tong, T. Y. N., Forouhi, N. G., Khandelwal, S., Prabhakaran, D., . . . de Lorgeril, M. (2014). Definitions and potential health benefits of the Mediterranean diet: Views from experts around the world. BioMed Central Medicine, 12(1). doi:10.1186/1741-7015-12-112.

Trichopoulou, A., Soukara, S., \& Vasilopoulou, E. (2007). Traditional foods: a science and society perspective. Trends in Food Science \& Technology, 18(8), 420-427. doi:10.1016/j.tifs.2007.03.007.

UNESCO. (2003). Convention for the Safeguarding of the Intangible Cultural Heritage. Retrieved from Paris: https://ich.unesco.org/en/convention.

UNESCO. (2013). Mediterranean diet. Retrieved from http://www.unesco.org/culture/ich/en/RL/mediterraneandiet-00884

Valagão, M. M. (2015). Identidade alimentar mediterrânica de Portugal e do Algarve. In U. d. Algarve (Ed.), Dimensões da Dieta Mediterrânica: Património Cultural Imaterial da Humanidade (pp. 301).

Vilarnau, C., Stracker, D. M., Funtikov, A., da Silva, R., Estruch, R., \& Bach-Faig, A. (2019). Worldwide adherence to Mediterranean Diet between 1960 and 2011. European Journal of Clinical Nutrition, 72 , 83-91. doi:10.1038/s41430-018-0313-9

WHO. (1946). Preamble to the Constitution of WHO as adopted by the International Health Conference, 19 June - 22 July 1946; signed on 22 July 1946 by the representatives of 61 States (Official Records of WHO, no. 2, p. 100) and entered into force on 7 April 1948. New York.

Willett, W. C., Sacks, F., Trichopoulou, A., Drescher,
G., Ferro-Luzzi, A., Helsing, E., \& Trichopoulos, D. (1995). Mediterranean diet pyramid: a cultural model for healthy eating. American Journal of Clinical Nutrition, 61(6 Suppl), 1402s-1406s.

Wirfalt, E., Drake, I., \& Wallstrom, P. (2013). What do review papers conclude about food and dietary patterns? Food \& Nutrition Research, 57. doi:10.3402/fnr. v57i0.20523. 